\title{
Natural Examples of Quasivarieties With EDPM
}

\author{
Keith A. Kearnes
}

A quasivariety $\mathcal{K}$ has equationally definable principal meets, or EDPM, if there are finitely many pairs of terms $\left(p_{i}(x, y, z, u), q_{i}(x, y, z, u)\right), i<n$, such that for any $\mathbf{A} \in \mathcal{K}$ and $a, b, c, d \in \mathbf{A}$ we have

$$
\theta_{\mathcal{K}}(a, b) \cdot \theta_{\mathcal{K}}(c, d)=\theta_{\mathcal{K}}\left(\left\{\left(p_{i}(a, b, c, d), q_{i}(a, b, c, d)\right) \mid i \in I\right\}\right) .
$$

Here $\theta_{\mathcal{K}}(X)$ denotes the least congruence $\theta$ containing $X$ such that $\mathbf{A} / \theta \in \mathcal{K}$. Quasivarieties with EDPM have recently arisen in the study of finitely based quasivarieties. They are known to be relatively congruence distributive, which means that the lattice of congruences on $\mathbf{A}$ of the form $\theta_{\mathcal{K}}(X)$ is a distributive lattice for any $\mathbf{A} \in \mathcal{K}$.

Few examples of quasivarieties with EDPM which did not lie in a congruence distributive variety (or at least a modular variety) were known until it was proved in [2] that any finite order-primal algebra generates a relatively distributive quasivariety. (An algebra $\mathbf{A}$ is order-primal if there exists a partial ordering, $\langle A, \leq\rangle$, of the universe of $\mathbf{A}$ such that the terms of $\mathbf{A}$ are precisely the operations on $A$ which are monotone with respect to $\leq$.) Since an order-primal algebra $\mathbf{A}$ has no non-trivial subalgebras, the only finitely subdirectly irreducible algebra in $\mathbf{S P}(\mathbf{A})$ up to isomorphism is $\mathbf{A}$. The class of finitely subdirectly irreducible algebras forms a universal class, so it follows from Theorem $2.3(i) \leftrightarrow(i v)$ of [1] that the quasivariety generated by a finite order-primal algebra has EDPM. The result in [2], that finite order-primal algebras generate relatively distributive quasivarieties, uses a fairly long argument involving generalized duality theory. The same result was later proved in [3] using tame congruence theory. We now give a short, direct proof that a certain class of finite algebras (including all the order-primal algebras) generate quasivarieties with EDPM.

Theorem If $\mathbf{A}$ is a finite algebra and $\langle A, \leq\rangle$ is a partial order such that every 4-ary operation on $A$ which is monotone with respect to $\leq$ is a term of $\mathbf{A}$, then $\mathcal{K}=\mathbf{S P}(\mathbf{A})$ is a quasivariety with EDPM. Hence, $\mathcal{K}$ is a relatively distributive quasivariety.

Proof: If A satisfies the hypotheses of the Theorem, then $\mathbf{A}$ is subdirectly irreducible and has no non-trivial subalgebras. It is easy to prove (and this result is Theorem $2.3(i) \leftrightarrow$ (vii) of [1]) that the pairs $\left(p_{i}, q_{i}\right), i<n$, are terms witnessing EDPM for $\mathcal{K}$ iff

$$
\mathbf{A} \models \forall x, y, z, u\left(\bigwedge_{i<n}\left(p_{i}(x, y, z, u)=q_{i}(x, y, z, u)\right) \Leftrightarrow x=y \text { or } z=u\right) .
$$

If we let $I=\left\{(a, b, c, d) \in A^{4} \mid a \neq b\right.$ and $\left.c \neq d\right\}$ we may rewrite this as

$$
\mathbf{A} \models \forall \bar{x}\left(\bigwedge_{i<n}\left(p_{i}(\bar{x})=q_{i}(\bar{x})\right) \Leftrightarrow \bar{x} \notin I\right) .
$$

We will construct such pairs $\left(p_{i}, q_{i}\right)$. 
We may assume that $\mathbf{A}$ is non-trivial. If no two elements of $\mathbf{A}$ are $\leq$-comparable, then every binary operation on $A$ is a term of $\mathbf{A}$. A classical result of Sierpinski implies that every finitary operation on $A$ is a term of $\mathbf{A}$. We can change the ordering on $A$ so that two elements are comparable and still retain the hypotheses of this theorem. Hence we may assume that $u \neq v$ are elements of $A$ such that $u \leq v$. Recall the definition of $I$. For each $i \in I$ we define functions $p_{i}, q_{i}: A^{4} \rightarrow A$ by:

$$
p_{i}(\bar{x})=\left\{\begin{array}{ll}
u & \text { if } \bar{x} \leq i \\
v & \text { otherwise }
\end{array} \quad \text { and } q_{i}(\bar{x})= \begin{cases}p_{i}(\bar{x}) & \text { if } \bar{x} \neq i \\
v & \text { if } \bar{x}=i\end{cases}\right.
$$

The order on $A^{4}$ in this definition is the product order. $p_{i}$ and $q_{i}$ are monotone, therefore equal to terms, and $p_{i}(\bar{x})=q_{i}(\bar{x})$ iff $\bar{x} \neq i$. Hence,

$$
\mathbf{A} \models \forall \bar{x}\left(\bigwedge_{i \in I}\left(p_{i}(\bar{x})=q_{i}(\bar{x})\right) \Leftrightarrow \bar{x} \notin I\right) .
$$

Since $|I|$ is finite, we have shown that $\mathcal{K}$ has EDPM. By Theorem 2.3 of $[1]$, the fact that $\mathcal{K}$ is relatively congruence distributive follows from the fact that it has EDPM.

Corollary Every finite order-primal algebra generates a quasivariety with EDPM.

It is known that not every order-primal algebra lies inside a modular variety. The order primal algebras that lie inside a modular variety are characterized in [3].

\section{References}

[1] J. Czelakowski and W. Dziobiak, Congruence distributive quasivarieties whose finitely subdirectly irreducible members form a universal class, Algebra Universalis 27 (1990), 128-149.

[2] B. Davey, R. W. Quackenbush, and D. Schweigert, Monotone clones and the varieties they determine, (preprint).

[3] R. McKenzie, Monotone clones, residual smallness and congruence distributivity, Bull. Austral. Math. Soc. 41 (1990), 283-300.

1980 Mathematics subject classification: 08B10, 08C15.

University of Hawaii, Honolulu HI 96822 